\title{
Eigensolution to Morse potential for Scandium and Nitrogen monoiodides
}

\author{
C. A. Onate ${ }^{a, b, *}$, G. O. Egharevba ${ }^{a}$, D. T. Bankole ${ }^{a}$ \\ ${ }^{a}$ Department of Physical Sciences, Landmark University, Omu-Aran, Nigeria. \\ ${ }^{b}$ Landmark University SDG 4 (Quality Education)
}

\begin{abstract}
The solutions for Morse potential energy function under the influence of Schrödinger equation are examined using supersymmetric approach. The energy equation obtained was used to generate eigenvalues for $X^{1} \Sigma^{+}$state of scandium monoiodide (ScI) and $X^{3} \sum^{-}$state of nitrogen monoiodide (NI) respectively by imputing their respective spectroscopic parameters. The calculated results for the two molecules aligned excellently with the predicted/observed values.
\end{abstract}

DOI:10.46481/jnsps.2021.407

Keywords: Eigensolutions, Wave equation, Bound state, Molecules

Article History :

Received: 13 September 2021

Received in revised form: 27 September 2027

Accepted for publication: 28 October 2021

Published: 29 November 2021

(c)2021 Journal of the Nigerian Society of Physical Sciences. All rights reserved. Communicated by: W. A. Yahya

\section{Introduction}

The study of the interactions for atomic molecules can be carried out using some physical potential models. Over the years, the energy eigenvalues of different molecules were obtained for different potential terms and reported. Among the reported works are, the energy eigenvalues of four molecules for Kratzer potential by Bayrak et al. [1]. In ref. [2], Ikhdair obtained energy eigenvalues of eight molecules for ManningRosen potential. Ikhdair and Sever [3], obtained energy eigenvalues of six molecules for Kratzer-type potential, Falaye et al. [4], under the study of Tietz-Wei diatomic molecular potential function, obtained energy eigenvalues of ten molecules. In ref. [5], the energy eigenvalues of some molecules were obtained for pseudoharmonic potential. Onate et al. [6], obtained energy eigenvalues of six molecules for hyperbolic-sinus

${ }^{*}$ Corresponding author tel. no:

Email address: oaclems14@physicist.net (C. A. Onate) potential model. Recently, Farout et al. [7], obtained exact momentum states of some molecules for improved deformed exponential-type potential. Despite all the reports given above, it has not been easy to deduce a potential energy for various molecules, thus, a challenging issue subsist in the study. However, some potential energy function that can explain various diatomic molecules in good agreement with experimental data have been proposed based on the experimental constants such as the dissociation energy and equilibrium bond length, e.g. Deng-Fan, Improved Rosen-Morse, Morse, Tietz-Hua oscillator, improved generalized Poschl-Teller oscillator [8-13]. The energies of these potential functions were calculated for cesium molecule, sodium dimer, nitrogen dimer, hydrogen molecule and potassium. The result obtained for each molecule aligned with the experimental data. However, it is noted that only few potential functions were examined and their results compared with the experimental results. Motivated by the interest in the Morse potential function as one of the different potential en- 


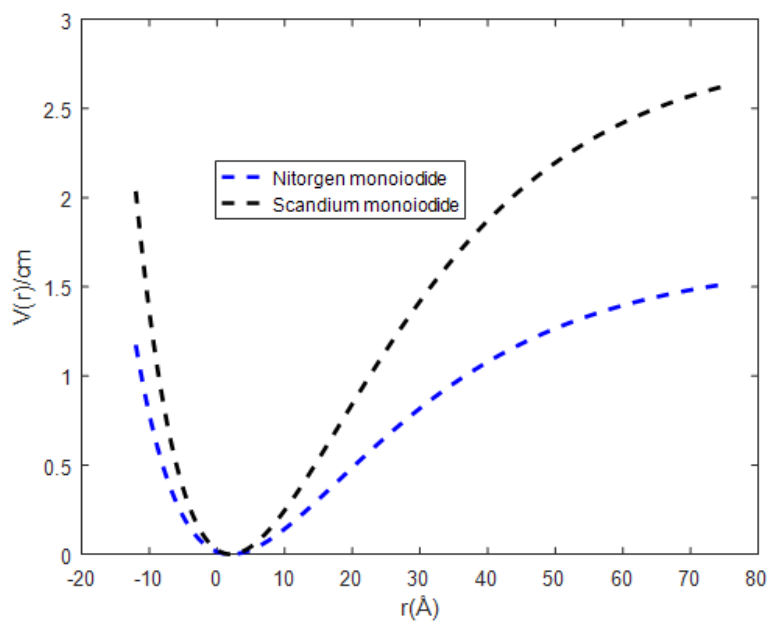

Figure 1: The Morse potential function for scandium monoiodide and nitrogen monoiodide

ergy functions suggested to obtain information about diatomic molecular structures, this work aims to determine the energy eigenvalues of scadium monoiodide and nitrogen monoiodide for a Morse potential energy function. The Morse potential model is a molecular potential model that is used to describe the interaction between two atoms in the atomic domain, as well as interaction closed to the surface. Several works have been reported on the Morse potential with different methods. However, most of the reports given are on the Morse potential of the form [17]

$$
V(r)=-2 D_{e} e^{-\alpha\left(r-r_{e}\right)}+D_{e}^{-2 \alpha\left(r-r_{e}\right)},
$$

where $D_{e}$ is the dissociation energy, $r_{e}$ is the equilibrium bond length, $r$ is the internuclear separation and $\alpha$ is a screening parameter. According to Akanbi et al. [18], the Morse potential given above has a minimum value at $r=r_{e}$ and it is zero at $r=\infty$. The authors further emphasized that the barrier is assumed to be outside the influence of the Morse oscillator. In refs. [19], another form of Morse potential called Shifted Morse was studied. The form of Morse potential is given as

$$
V(r)=(\ell+1)^{2}-(2 \ell+3) e^{-x}+e^{-2 x} .
$$

In the present study, the interacting Morse potential

$$
V(r)=D_{e}\left(1-2 e^{-\alpha\left(r-r_{e}\right)}+e^{-2 \alpha\left(r-r_{e}\right)}\right)
$$

will be considered. The form of Morse potential model was studied by Desai et al. [20] in one of their papers. However, the explicit detail analysis for the solutions of the Morse potential as well as the energy equation were not given. In this study, the analysis of eigenvalues and eigenfunctions for the Morse potential will be given in detail. Figure 1 below, depicts the shape of Morse potential function for scandium monoiodide (ScI) and nitrogen monoiodide (NI).

\section{Bound state solution of the Schrödinger equation using supersymmetric approach}

Here, the eigenvalue and eigenfunction for the Morse potential is obtained. The radial Schrödinger equation with an interacting potential $V(r)$ is given by

$$
\left[-\frac{\hbar^{2}}{2 \mu} \frac{d^{2}}{d r^{2}}-E_{n, \ell}+V(r)\right] R_{n, \ell}(r)=0,
$$

where $E_{n, \ell}$ is the non-relativistic energy of the system, $\hbar$ is a reduced Planck's constant, $\mu$ is the reduced mass, $R_{n, \ell}(r)$ is the wave function. The solutions of Eq. (2) can be obtained using different traditional methodologies. In this work, as said earlier, the supersymmetric approach will be used for the calculation. The supersymmetric approach is one of the approximate methods used to solve wave equations. The method depends on the proposition of superpotential. To use this method, first we plug Eq. (3) into Eq. (4) to have

$$
\begin{aligned}
& \frac{d^{2} R_{n}(r)}{d r^{2}} \\
& =\frac{2 \mu\left(D_{e}-E_{n}-2 D_{e} e^{-\alpha\left(r-r_{e}\right)}+D_{e} e^{-2 \alpha\left(r-r_{e}\right)}\right)}{\hbar^{2}} R_{n}(r)=0 .
\end{aligned}
$$

To solve the equation above using supersymmetric and shape invariance approach [21-23], the next step is to write a ground state wave function

$$
R_{0}(r)=\exp \left(-\int U(r) d r\right)
$$

where, $U(r)$ is the superpotential function. Invoking Eq. (6) onto Eq. (5) gives another relation satisfied by the superpotential function $U(r)$ :

$$
\begin{aligned}
U^{2}(r) & -\frac{d U(r)}{d r} \\
& =\frac{2 \mu\left(D_{e}-E_{n}\right)}{\hbar^{2}}+\frac{2 \mu\left(D_{e} e^{-\alpha\left(r-r_{e}\right)}-2 D_{e}\right)}{\hbar^{2}} e^{-\alpha\left(r-r_{e}\right)}
\end{aligned}
$$

To validate the compatibility of the two sides of Eq. (7) [24], we express the superpotential function as

$$
U(r)=\rho_{0}-\rho_{1} e^{-\alpha r}
$$

The two terms $\rho_{0}$ and $\rho_{1}$ in Eq. (8) are superpotential constants and their respective values will soon be determined. Plugging Eq. (8) into Eq. (7) with some mathematical manipulations and simplifications leads to the following reasonable equations

$$
\begin{aligned}
& \rho_{0}^{2}=\frac{2 \mu D_{e}}{\hbar^{2}}-\frac{2 \mu E_{n}}{\hbar^{2}}, \\
& \rho_{1}=\sqrt{\frac{2 \mu D_{e} e^{2 \alpha r_{e}}}{\hbar^{2}}}, \\
& \rho_{0}=\frac{\frac{4 \mu D_{e} e^{\alpha_{e}}}{\hbar^{2}}-\alpha \rho_{1}}{2 \rho_{1}} .
\end{aligned}
$$

We consider the bound state solutions that demand the wave function $R_{n}(r)$ which satisfy the boundary conditions for $R_{n}(\infty)=$ 
0and $R_{n}(0)$ is limitary. These regularity conditions suggest that both $\rho_{0}$ and $\rho_{1}$ are greater than zero. Having determined the two superpotential constants, the supersymmetric partner potentials $U^{2}(r) \pm \frac{d U(r)}{d r}$ can easily be constructed as follows

$$
\begin{aligned}
& V_{+}(r)=U^{2}(r)+\frac{d U(r)}{d r}=\rho_{0}^{2}-\rho_{1}(2 A-\alpha) e^{-\alpha r}+\rho_{1}^{2} e^{-2 \alpha r}, \\
& V_{-}(r)=U^{2}(r)-\frac{d U(r)}{d r}=\rho_{0}^{2}-\rho_{1}(2 A+\alpha) e^{-\alpha r}+\rho_{1}^{2} e^{-2 \alpha r} .
\end{aligned}
$$

Eq. (12) and Eq. (13) are related by a simple relation

$$
V_{+}\left(r, a_{0}\right)=V_{-}\left(r, a_{1}\right)+R\left(a_{1}\right),
$$

where $a_{0}$ is an old set of parameters and $a_{1}$ is a new set of parameters uniquely determined from the old set of parameters. The term $R\left(a_{1}\right)$ is called a reminder term that do not dependent of the variable $r$. In the concept of shape invariance potential, $a_{0}=\rho_{0}$ as $\rho_{0} \rightarrow \rho_{0}+\alpha n$. Using the partner potential $V_{-}\left(r, a_{1}\right)$, Eq. (9), Eq. (10) and Eq. (11), the energy of the Morse potential function can be obtain following

$$
\begin{gathered}
E_{n}^{(-)}=\sum_{k=1} R\left(a_{k}\right)=R\left(a_{1}\right)+R\left(a_{2}\right)+R\left(a_{3}\right) \\
+\ldots \ldots+R\left(a_{n-1}\right)+R\left(a_{n}\right)=\left(\frac{\rho_{1}-\alpha a_{0}}{2 a_{0}}\right)^{2}+\left(\frac{\rho_{1}-\alpha a_{1}}{2 a_{1}}\right)^{2} \\
\quad-\left(\frac{\rho_{1}-\alpha a_{1}}{2 a_{1}}\right)^{2}+\left(\frac{\rho_{1}-\alpha a_{2}}{2 a_{2}}\right)^{2} \\
-\left(\frac{\rho_{1}-\alpha a_{2}}{2 a_{2}}\right)^{2}+\left(\frac{\rho_{1}-\alpha a_{3}}{2 a_{3}}\right)^{2}-\left(\frac{\rho_{1}-\alpha a_{3}}{2 a_{3}}\right)^{2}+\left(\frac{\rho_{1}-\alpha a_{4}}{2 a_{4}}\right)^{2}+\ldots . \\
\left(\frac{\rho_{1}-\alpha a_{n-1}}{2 a_{n-1}}\right)^{2}+\left(\frac{\rho_{1}-\alpha a_{n}}{2 a_{n}}\right)^{2}=\left(\frac{\rho_{1}-\alpha a_{0}}{2 a_{0}}\right)^{2}+\left(\frac{\rho_{1}-\alpha a_{n}}{2 a_{n}}\right)^{2} \\
=\left(\frac{4 \mu D_{e} e^{\alpha r_{e}}-\alpha a_{0}}{2 a_{0}}\right)^{2}+\left(\frac{4 \mu D_{e} e^{\alpha r_{e}}-\alpha a_{n}}{2 a_{n}}\right)^{2}
\end{gathered}
$$

Following the formalism of supersymmetric approach, the complete energy equation is obtain as

$$
E_{n}=D_{e}-\frac{\alpha^{2} \hbar^{2}}{2 \mu}\left[\frac{\frac{2 \mu D_{e} e^{\alpha r_{e}}}{\alpha^{2} \hbar^{2}}-\left(n+\frac{1}{2}\right) \sqrt{\frac{2 \mu D_{e} e^{2 \alpha r_{e}}}{\alpha^{2} \hbar^{2}}}}{\sqrt{\frac{2 \mu D_{e} e^{22 r_{e}}}{\alpha^{2} \hbar^{2}}}}\right]^{2} .
$$

\subsection{Wave Function}

To obtain the wave function, we make a transformation of the form $y=\frac{1}{e^{\alpha r}}$ and invoke it on Eq. (5) to have

$$
\frac{d^{2} R_{n}(y)}{d y^{2}}-\frac{1}{y} \frac{d R_{n}(y)}{d y}+\frac{\frac{2 \mu\left[E_{n}-D_{e}+D_{e} e^{\alpha r e} y\left(2-e^{\alpha r e} y\right)\right]}{\alpha^{2} \hbar^{2}}}{y^{2}} R_{n}(y)=0
$$

Following the paper of Tezcan and Sever [25], the radial wave function for the Morse potential becomes

$$
R_{n}(y)=N y \sqrt{\frac{2 \mu\left(E_{n}-D_{e}\right)}{\alpha^{2} \hbar^{2}}} e^{-y} \sqrt{\frac{2 \mu D_{e} e^{2} \alpha_{e}}{\alpha^{2} \hbar^{2}}} L_{n}^{2 \sqrt{\frac{2 \mu\left(E_{n}-D_{e}\right)}{\alpha^{2} \hbar^{2}}}}\left(2 \sqrt{\frac{2 \mu\left(E_{n}-D_{e}\right)}{\alpha^{2} \hbar^{2}} y}\right) .
$$

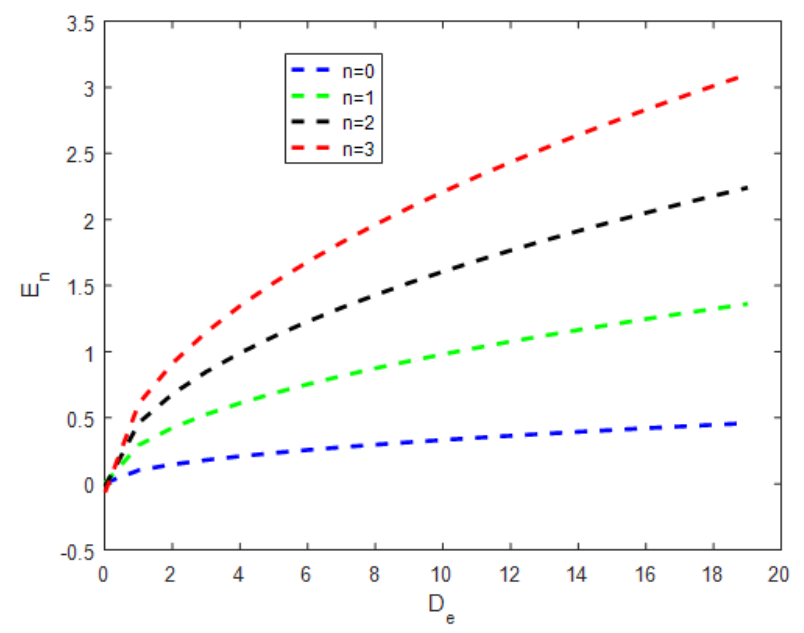

(a)

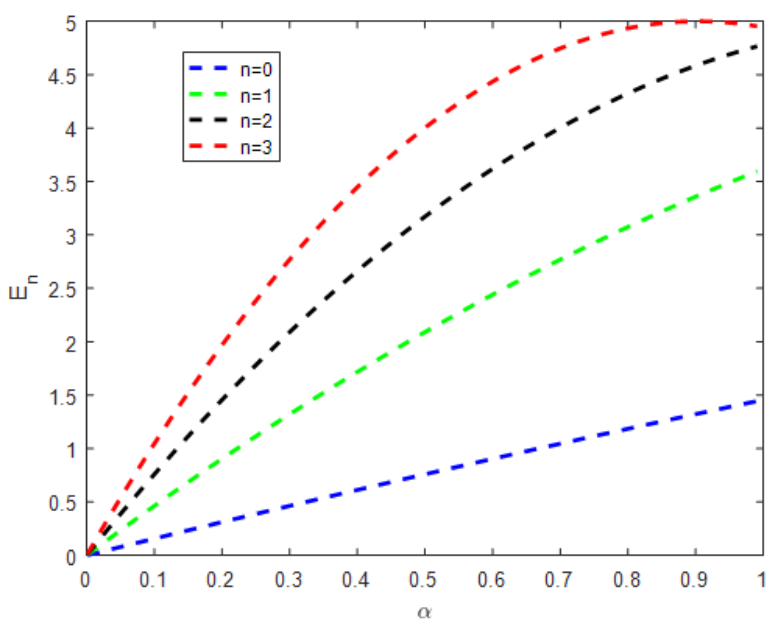

(b)

Figure 2: Variation of $E_{n}\left(\mathrm{~cm}^{-1}\right)$ against $D_{e}\left(\mathrm{~cm}^{-1}\right)$ and $\alpha$ respectively

\section{Discussion}

The shape of Morse potential for scandium monoiodide and nitrogen monoiodide is shown in Figure 1. In Figures 2 (a) and (b), the variation of energy against the dissociation energy and screening parameter respectively are shown. The energy and each of the dissociation energy and screening parameter respectively varies inversely with each other. In each case, the highest quantum state has the highest energy. The energies at various quantum state at $D_{e}$ and $\alpha=0$ respectively are zero, which is the point of convergence for the energies at different quantum states. The vibrational energies of the Morse potential for various values of the screening parameter, quantum number and dissociation energy are presented in Table 1 . The energy of the system rises with an increase in the quantum number, screening parameter and dissociation energy respectively. For a unity value of the dissociation energy, the energy of the system has turning point as the quantum number and the screening param- 
Table 1: Vibrational energies (in $\mathrm{cm}^{-1}$ ) of Morse potential model with $\mu=\hbar=1$ and $r_{e}=0.5 \dot{A}$ for three values of $D_{e}\left(\mathrm{~cm}^{1}\right)$ and various values of $n$ and $\alpha\left(\mathrm{cm}^{-1}\right)$.

\begin{tabular}{lll}
\hline$n$ & $\alpha$ & $E_{n}\left(D_{e}=1\right) E_{n}\left(D_{e}=5\right) E_{n}\left(D_{e}=10\right)$ \\
\hline 0 & 0.05 & 0.03504280 .07874440 .1114909 \\
& 0.25 & 0.16896420 .38747220 .5512045 \\
& 0.55 & 0.35109620 .83181371 .1920249 \\
& 0.75 & 0.46001761 .11554161 .6067385 \\
& 0.05 & 0.10325350 .23435830 .3325977 \\
& 0.25 & 0.46001761 .11554161 .6067385 \\
& 0.55 & 0.82641372 .26856663 .3491997 \\
2 & 0.75 & 0.95817782 .92474994 .3983404 \\
& 0.05 & 0.16896420 .38747220 .5512045 \\
& 0.25 & 0.68857101 .78111102 .5997725 \\
& 0.55 & 0.99923123 .40281935 .2038744 \\
3 & 0.75 & 0.89383794 .17145816 .6274424 \\
& 0.05 & 0.23217490 .53808610 .7673113 \\
& 0.25 & 0.85462442 .38418053 .5303065 \\
& 0.55 & 0.86954864 .23457206 .7560492 \\
& 0.75 & 0.26699814 .85566648 .2940444 \\
\hline
\end{tabular}

Table 2: Comparison of the calculated energies (incm ${ }^{-1}$ ) for $X^{1} \Sigma^{+}$state of ScI and $X^{3} \sum^{-}$state of NI with the predicted experimental RKR values of the Morse potential function

\begin{tabular}{ccccccc}
\hline$n$ & & $S c I$ & & & $N I$ & \\
& Calculated & {$[27]$} & LTE & calculated & {$[27]$} & LTE \\
\hline 0 & 138.4121 & 138.3 & 0.1121 & 301.6 & 301.1 & 0.5000 \\
1 & 414.7976 & 413.9 & 0.4976 & 898.0135 & 896.6 & 1.4135 \\
2 & 688.6233 & 687.7 & 0.9233 & 1486.4617 & 1482.3 & 3.1617 \\
3 & 961.1402 & 959.9 & 1.2402 & 2062.5594 & 2058.8 & 3.7594 \\
4 & 1232.1101 & 1230.4 & 1.7101 & 2629.9899 & 2625.9 & 4.0899 \\
5 & 1501.6044 & 1499.3 & 2.3044 & 3187.9671 & 3183.6 & 4.3671 \\
6 & 1769.1278 & 1766.5 & 2.6278 & 3736.8788 & 3731.9 & 4.9788 \\
\hline
\end{tabular}

eter increases respectively.

The observed data and the calculated energies for $X^{1} \Sigma^{+}$state of ScI and $X^{3} \Sigma^{-}$state of NI obtained using Eq. (15) are given in Table 2. The relative deviation LTE (calculated result minus experimental result) of the calculated results from the experimental results are also given in Table 2. The experimental values used for this work are taken from ref. [27]. For ScI, $D_{e}=2.858 \mathrm{eV}, r_{e}=2.6708 \AA, \omega_{e}=277.18 \mathrm{~cm}^{-1}$ while that of NI are $D_{e}=1.648 \mathrm{eV}, r_{e}=1.9653 \AA, \omega_{e}=604.7 \mathrm{~cm}^{-1}$. The screening parameter is calculated using

$$
\alpha=\pi c \omega_{e} \sqrt{\frac{2 \mu}{D_{e}}} .
$$

It is shown that the relative deviation becomes larger as the vibrational quantum state increases for the two molecules. However, the relative deviation for $X^{3} \sum^{-}$state of NI are higher compared to the relative deviation for $X^{1} \sum^{+}$state of ScI. This simply means that the ScI are more fitted for the calculation compared to NI. The average absolute percentage deviation for each of the molecule is calculated using the formula

$$
\sigma_{a v}=\frac{100}{N} \sum_{v}\left|\frac{E_{V}-E_{R K R}}{E_{R K R}}\right| .
$$

where $E_{R K R}$ is the observed values, $E_{V}$ is the present results and is the number of observed data points. The formula seems to be the revised version of what is given in ref. [18]. The calculated results in this study are greater than the experimental values, thus, to avoid negative deviation, we revised the order of the both the experimental and calculated values. The average absolute percentage deviation for $\mathrm{ScI}$ is $0.021 \%$ while that of NI is $0.032 \%$. To determine the proximity of the present results to the predicted values, the percentage error for each of the calculated result is computed using the formula

$$
\operatorname{err}_{p}=\frac{\sum_{v} L T E}{E_{R K R}} \times 100 .
$$

The percentage error for the results of ScI is $0.14 \%$ while that of the NI is $0.23 \%$.

\section{Conclusion}

In the present study, we calculated the energies of ScI and NI for a Morse potential function. The computed results aligned excellently with the observed results for the two molecules. The computation of the percentage error shows that the Morse potential is more fitted in the computation for ScI than NI since the percentage error in $\mathrm{ScI}$ is smaller than that of NI.

\section{References}

[1] O. Bayrak, I. Boztosun \& H. Ciftci, "Exact analytical solutions of the Kratzer potential by the asymptotic iteration method", International Journal of Quantum Chemistry 107 (2007) 540.

[2] S. M. Ikhdair, "On the bound state solutions of the Manning-Rosen potential including an improved approximation to the orbital centrifugal term", Physica Scripta 83 (2011) 10.

[3] S. M. Ikhdair \& R. Sever, "Exact quantization rule of the Kratzer-type potentials: An application to the diatomic molecules", Journal of Mathematical Chemistry 45 (2009) 1137.

[4] B. J. Falaye, K. J. Oyewumi, S. M. Ikhdair \& M. Hamzavi, "Eigensolution techniques, their applications and Fisher's information entropy of the Tietz-Wei diatomic molecular model", Physica Scripta 89 (2014) 115204.

[5] K. J. Oyewumi \& K. D. Sen, "Exact solutions of the Schrodinger equation for the pseudoharmonic potential: an application to some diatomic molecules", Journal of Mathematical Chemistry 50 (2012) 1039.

[6] C. A. Onate, A. Abolarinwa, S. O. Salawu \& N. K. Oladejo, "Bound state solutions of the Schrodinger equation and its application to some diatomic molecules", Journal of Molecular Modeling 26 (2020) 145.

[7] M. Farout, A. Bassalat \& S. M. Ikhdair, "Exact quantizated momentum eigenvalues and eigenstates of a general potential model", Journal of Applied Mathematical Physics 8 (2020) 1434

[8] R. Horchani, H. Jelassil, A. N. Ikot \& U. S. Okorie, "Rotation vibration spectrum of potassium molecules via the improved generalized PöschlTeller oscillator", International Journal of Quantum Chemistry 2020; e26558.

[9] S. Kaur \& C. G. Mahajan, "Some new four-parameter potentials and their use in the study of vibrational thermodynamic quantities of diatomic molecules", Pramana Journal of Physics 52 (1999) 459.

[10] J. Y. Liu, X.-T. Hu \& C.-S. Jia, "Molecular energies of the improved Rosen-Morse potential energy model", Canadian Journal of Chemistry 95 (2014) 40.

[11] C. A. Onate \& T. A. Akanbi, "Solutions of the Schrodinger equation with improved Rosen Morse potential for nitrogen molecule and sodium dimer", Results in Physics 22 (2021) 103961

[12] C. A. Onate, T. A. Akanbi \& I. B. Okon, "Ro-vibrational energies of cesium dimer and lithium dimer with molecular attractive potential", Scientific Reports 11 (2021) 6198. 
[13] C. A. Onate, M. C. Onyeaju, E. Omugbe, I. B. Okon \& O. E. Osafile, "Bound-state solutions and thermal properties of the modified Tietz-Hua potential", Scientific Reports 11 (2021) 2129.

[14] X. T. Hu, J.-Y. Liu \& C.-S. Jia, "The $3^{3} \sum_{g}^{+}$state of $\mathrm{Cs}_{2}$ molecule", Computational and Theoretical Chemistry 1019 (2013) 137.

[15] M. L. da Silva, V. Guerra, L. Loureiro \& P. A. Sa, "Vibrational distributions in N2 with an improved calculation of energy levels using the RKR method", Chemical Physics 348 (2008) 187.

[16] R. Horchani, N. Al-Kinda \& H. Jelassi, "Ro-vibrational energies of caesium molecules with the Tietz-Hua oscillator", Molecular Physics (2020) e1812746.

[17] D. A. Morales, "Supersymmetric improvement of the Pekeris approximation for the rotating Morse potential", Chemical Physics Letters 394 (2004) 68.

[18] T. A. Akanbi, C. A. Onate \& O. Y. Oludoun, "Eigensolutions and thermodynamic properties of deformed and modified Morse potential model", AIP Advances 11 (2021) 045213.

[19] M. Zarezadeh \& M. K. Tavassoly, "The Solution of the Schrodinger equation for a particular form of Morse Potential by using Laplace Transform", CPC (HEP \& NP) 33 (2009) 1.

[20] A. M. Desai, N. Mesquita \& V. Fernandes, "A new modified Morse potential energy function for diatomic molecules", Physica Scripta 95 (2020) 085401
[21] E. Witten, "Dynamical Breaking of Supersymmetry", Nuclear Physics B 188 (1981) 513

[22] H. Hassanabadi, E. Maghsoodi, S. Zarrinkamar \& H. Rahimov, "An Approximate solutions of the Dirac Equation for Hyperbolic scalar and vector potentials and a Coulomb tensor interaction by SUSY QM", Modern Physics Letters A 26 (2011) 2703.

[23] F. Cooper, A. Khare \& U. Sukhatme, "Supersymmetry in Quantum Mechanics", Physical Reports 251 (1995) 267.

[24] C. A. Onate, "Relativistic and non-relativistic solutions of the inversely quadratic Yukawa Potential”, African Review of Physics 8 (2013) 325.

[25] C. Tezcan \& R. Sever, "A general approach for the exact Solution of the Schrödinger equation", International Journal of Theoretical Physics 48 (2009) 337.

[26] C. A. Onate, M. C. Onyeaju, A. N. Ikot, J. O. A. Idiodi \& J. O. Ojonubah, "Eigen Solutions, Shannon Entropy and Fisher Information under the Eckart Manning Rosen Potential Model", Journal of the Korean Physical Society 70 (2017) 339

[27] R. R. Reddy, Y. N. Ahammed, B. S. Devi, P. A. Azeem, K. R. Gopal \& T. V. R. Rao, "Potential energy curves, dissociation energies and Franck Condon factors for NI and ScI molecules", Journal of Quantum Spectrosc andopy Radiation Transfer 74 (2002) 125 